\title{
IL-21 and probiotic therapy improve Th17 frequencies, microbial translocation, and microbiome in ARV-treated, SIV-infected
} macaques

\author{
AM Ortiz ${ }^{1}$, ZA Klase ${ }^{1,2}$, SR DiNapoli ${ }^{1}, I_{\text {Vujkovic-Cvijin }}{ }^{3}, \mathrm{~K}_{\text {Carmack }}{ }^{1}, \mathrm{MR}_{\text {Perkins }}{ }^{1}, \mathrm{~N}_{\text {Calantone }}{ }^{1}$, \\ CL Vinton ${ }^{1}$, NE Riddick ${ }^{1}$, J Gallagher ${ }^{1}$, NR Klatt ${ }^{1,4}$, JM McCune ${ }^{3}$, JD Estes ${ }^{5}, M$ Paiardini $^{6}$ and \\ JM Brenchley ${ }^{1}$
}

Increased mortality in antiretroviral (ARV)-treated, HIV-infected individuals has been attributed to persistent immune dysfunction, in part due to abnormalities at the gastrointestinal barrier. In particular, the poor reconstitution of gastrointestinal Th17 cells correlates with residual translocation of dysbiotic, immunostimulatory microflora across a compromised intestinal epithelial barrier. We have previously demonstrated that oral probiotics promote increased intestinal CD4 ${ }^{+}$T-cell reconstitution during ARV treatment in a non-human primate model of HIV infection; however, essential mucosal T-cell subsets, such as Th17 cells, had limited recovery. Here, we sought to promote Th17 cell recovery by administering interleukin (IL)-21 to a limited number of ARV-treated, probiotic-supplemented, Simian Immunodeficiency Virus (SIV)-infected pigtailed macaques. We demonstrate that probiotic and IL-21 supplementation of ARVs are associated with enhanced polyfunctional Th17 expansion and reduced markers of microbial translocation and dysbiosis as compared with infected controls receiving ARVs alone. Importantly, treatment resulted in fewer morbidities compared with controls, and was independent of increased immune activation or loss of viral suppression. We propose that combining ARVs with therapeutics aimed at restoring intestinal stasis may significantly improve disease prognosis of ARV-treated, HIV-infected individuals.

\section{INTRODUCTION}

Progressive HIV infection is characterized by persistent innate and adaptive immune activation that is predictive of disease progression and that is not resolved with effective antiretroviral (ARV) therapy. ${ }^{1,2}$ Although mediators of immune activation are multi-faceted, it is evident that even with effective viral suppression, persistent immune dysfunction at the intestinal epithelial barrier permits the translocation of immunostimulatory luminal contents into intestinal tissues and systemic circulation. ${ }^{3}$ In non-human primate models of
HIV infection, microbial translocation distinguishes progressive from non-progressive models of Simian Immunodeficiency Virus (SIV) infection, with progressive infection models such as rhesus (Macaca mulatta) and pigtailed (Macaca nemistrina) macaques displaying evidence of microbial translocation and immune activation in stark contrast to non-progressive hosts such as sooty mangabeys (Cercocebus atys). ${ }^{4-6}$ Importantly, although both progressive and nonprogressive infected hosts exhibit some loss of intestinal CD $4^{+}$ T cells, progressive models exhibit a preferential loss of Th17

\footnotetext{
${ }^{1}$ Program in Tissue Immunity and Repair and Immunopathogenesis Section, Lab of Molecular Microbiology, NIAID, NIH, Bethesda, Maryland, USA. ${ }^{2}$ Department of Biological Sciences, University of the Sciences, Philadelphia, Pennsylvania, USA. ${ }^{3}$ Division of Experimental Medicine, Department of Medicine, University of California, San Francisco (UCSF), San Francisco, California, USA. ${ }^{4}$ Department of Pharmaceutics, Washington National Primate Research Center, University of Washington, Seattle, Washington, USA. ${ }^{5}$ AIDS and Cancer Virus Program, Frederick National Laboratory for Cancer Research, Leidos Biomedical Research, Inc., Frederick, Maryland, USA and ${ }^{6}$ Division of Microbiology and Immunology, Yerkes National Primate Research Center, Department of Pathology and Laboratory Medicine, Emory University School of Medicine, Atlanta, Georgia, USA. Correspondence: JM Brenchley (jbrench|@niaid.nih.gov)
} 
cells, implicating Th17 cell loss as a primary contributor to microbial translocation and disease progression. ${ }^{6-8}$

Th17 cells are a subset of differentiated, interleukin (IL)-17expressing memory CD4 ${ }^{+}$T cells important for control of the microbiota at mucosal barrier sites. ${ }^{9}$ Th17 cells develop from naïve $\mathrm{CD}^{+} \mathrm{T}$ cells in response to TGF- $\beta$ and proinflammatory cytokine signaling induced by microbial antigens. ${ }^{10-12}$ Although Th17 differentiation can be calibrated by the microbiome, ${ }^{13,14}$ the effects of Th17 cells on gastrointestinal immunity extend beyond interplay with commensal species. Thus, while commensal species are sufficient for the generation of Th17 cells, ${ }^{13,14}$ the ability of Th17 cells to induce production of antimicrobial peptides and chemotactic cytokines from the intestinal epithelium contributes to resistance against colonization with Salmonella typhimurium, Streptococcus pneumoniae, and Candida albicans. ${ }^{8,9}$ Implicit in this counterregulation, microbial dysbiosis-an imbalance in the relative frequencies of commensal and pathogenic species-is associated with a dysregulation of Th17 frequencies and function. ${ }^{15}$ This, in turn, is implicated in inflammation in a multitude of chronic autoimmune and infectious diseases. In HIV-infected individuals and SIV-infected macaques, microbial dysbiosis has been described as a contributor to Th17 loss and microbial translocation, with some dysbiotic species shown to be enriched in both treated and untreated subjects, and to be associated with markers of disease progression. ${ }^{16,17}$ Given the contributions of dysbiotic and translocating bacterial species to disease progression in treated, HIV-infected individuals, we previously considered that augmenting ARV therapy in chronically SIVinfected macaques with probiotics might promote a restoration of the commensal microbiota, initiating a sequence of immune restorative processes. Although probiotic supplementation was associated with intestinal $\mathrm{CD}^{+}{ }^{+} \mathrm{T}$-cell restoration and enhanced Th17 functionality, we did not observe a significant improvement in the frequency of Th17 cells or was there any discernible impact on the relative frequencies of fecal microbial communities. $^{18}$

Subsequent to priming by APCs, Th17 development is augmented by autocrine production of the common $\gamma$-chain cytokine, IL-21. ${ }^{19,20}$ Although not essential for the differentiation of Th17 cells, ${ }^{14,21}$ blockade of IL-21 during Th17 development limits the expansion and functional capacity of Th17 cells. ${ }^{19,20}$ In progressive SIV infection, a paucity of IL-21-producing CD4 ${ }^{+} \mathrm{T}$ cells correlates with Th17 loss. ${ }^{22}$ Moreover, although IL-21 treatment during acute SIV infection has been shown to delay Th17 loss independent of ARV therapy, cessation of IL-21 administration results in a rapid decline in Th17 frequencies. ${ }^{23}$

Herein, we considered that augmenting ARV therapy with combination probiotic and IL-21 therapy might enhance the recovery and maintenance of Th17 cells in chronic SIV infection. As described, we observed that this regimen is associated with increased frequencies of intestinal polyfunctional Th17 cells as compared with ARV-only controls, and that supplementation is further associated with improvements in microbial translocation, microbial dysbiosis, and clinical status. Importantly, as our therapy did not promote immune

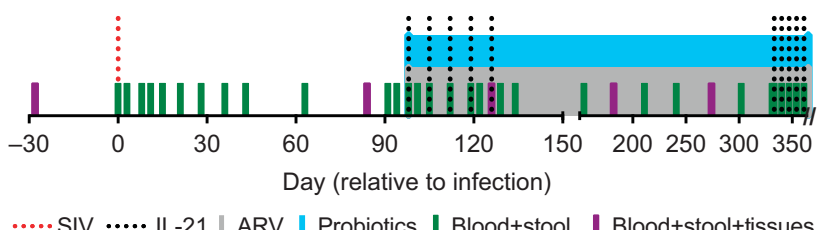

Figure 1 Experimental design. Eleven pigtailed macaques (PTMs) were infected with SIVmac $_{239}$ at day 0 (dotted red line) and daily antiretroviral (ARV) therapy (gray-shaded region) was initiated at day 98 p.i. Of these animals, 6 PTM were additionally treated with daily probiotic therapy (blueshaded region) as well as two rounds of IL-21 therapy, administered once weekly for 5 weeks (dotted black lines). One ARV-only PTM was euthanized at day 126 p.i. per protocol specified clinical end point. Blood, stool, and tissues (bronchoalveolar lavage (BAL), jejunal (Jej), lymph node $(\mathrm{LN})$, and rectal (RB) were collected at pre-determined intervals, as indicated (green and purple bars). SIV, Simian Immunodeficiency Virus.

activation or negate viral suppression, we believe that our findings suggest that probiotic and IL-21 supplementation of ARVs may translate into a safe and effective therapeutic for the reduction of co-morbidities suffered by ARV-treated, HIVinfected individuals.

\section{RESULTS}

\section{Experimental design}

We previously demonstrated that treatment of SIV-infected, virus-suppressed pigtailed macaques (PTM) with probiotics improved gastrointestinal integrity and immunity; however, probiotic supplementation alone did not promote a restoration of Th17 cells nor was there evidence for a shift toward a significantly less inflammatory microbiome. ${ }^{18}$ Here, we sought to determine whether treatment with IL-21 ${ }^{19,20}$ enhances probiotic treatment in SIV-infected, ARV-and probiotic-treated PTMs by promoting Th17 recovery and reducing microbial translocation and dysbiosis. As depicted in Figure 1, we infected 11 PTMs with SIV $_{\text {mac239 }}$ and, at day 98 post infection (p.i.), all animals began daily treatment with the reverse transcriptase inhibitors, tenofovir and emtricitabine, and the integrase inhibitor, L'812. Of these animals, six were simultaneously treated with the daily probiotic, VLS\#3 (consisting of $10^{11}$ live Bifidobacterium, Lactobacillus, and Streptococcus species) and with early and late administration courses of IL-21 to assess refractoriness to continued IL-21 therapy. We routinely sampled the blood and stool of all animals, sampled the pulmonary airways through bronchoalveolar lavage (BAL), and obtained jejunal (Jej), lymph node (LN), and rectal (RB) biopsies.

\section{IL-21 and probiotic supplementation does not alter viral suppression in ARV-treated macaques}

IL-21 is a pleiotropic cytokine known to promote the development of Th17 cells from naïve $\mathrm{CD}^{+}{ }^{+} \mathrm{T}$-cell precursors. ${ }^{19,20}$ As mature CD $4{ }^{+}$T cells are the preferential targets for HIV and SIV infection, we assessed the impact of the IL-21 and probiotic regimen on viral suppression. With the initiation of therapy, all animals exhibited a rapid decline in plasma viral RNA with all but one probiotic- and IL-21-supplemented animal (PTA2P031) achieving suppression to below 100 copies per $\mathrm{ml}$ within 30 days of treatment initiation (Figure 2a). In 

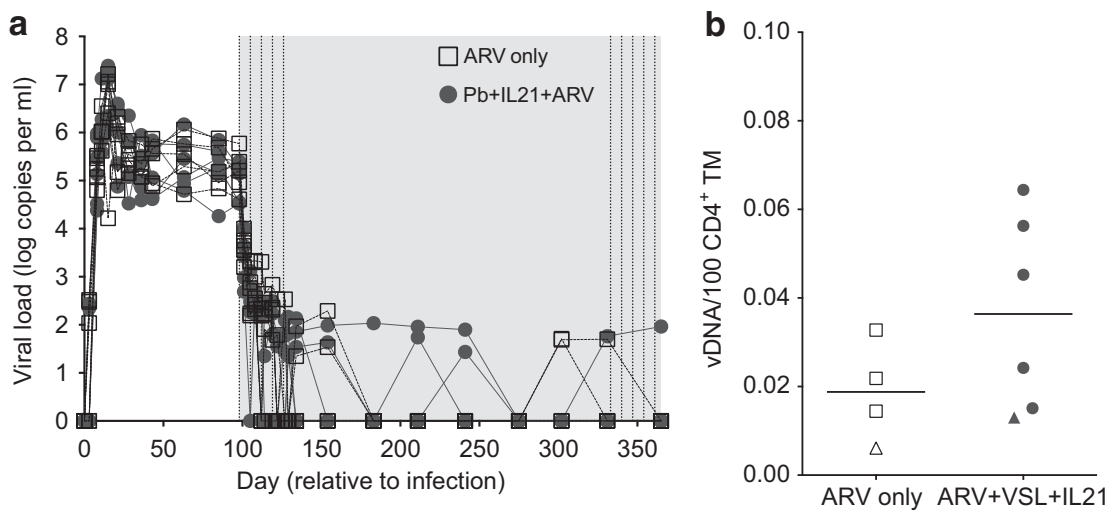

Figure 2 Interleukin (IL)-21 and probiotic therapy does not alter viral load or cellular distribution in antiretroviral (ARV)-treated pigtailed macaques (PTMs). (a) Longitudinal, log copies per ml of plasma viral RNA (vRNA) from animals treated with ARVs only (open squares) or with ARVs, probiotic VSL\#3, and IL-21 (closed circles). Gray shading indicates treatment initiation and duration. Dotted lines denote IL-21 administration time points. Symbols

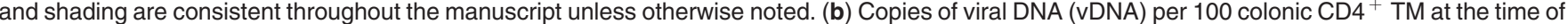
necropsy. Triangles indicate samples normalized to the limit of detection.

each group, all but one animal exhibited at least one instance of breakthrough viremia; however, measurable virus in these animals did not exceed 100 copies per ml. Importantly, breakthrough viremia in supplemented animals was not associated with increased activation of systemic $\mathrm{CD} 4^{+}$or $\mathrm{CD}^{+}{ }^{+}$memory T cells (TM), as assessed by HLA-DR and Ki67 expression, or with increases in plasma soluble CD14 (sCD14) or lipopolysaccharide (LPS)-binding protein (Supplementary Figure S1 online and data not shown).

IL-21 has been shown to promote the expansion of antiviral $\mathrm{CD}^{+}{ }^{\mathrm{T}}$ cells; $;^{23-25}$ however, we considered that $\mathrm{CD} 4^{+} \mathrm{T}$-cell reconstitution might counteract $\mathrm{ARV}$ - and $\mathrm{CD}^{+}{ }^{+} \mathrm{T}$-cellmediated antiviral effects by expanding the viral reservoir. Indeed, the addition of IL-21 to probiotic and ARV therapy was associated with enhanced intestinal $\mathrm{CD} 4{ }^{+} \mathrm{T}$-cell recovery as compared ARV-only controls (Supplementary Figure S2), similar to what we previously reported using probiotic supplementation alone. ${ }^{18}$ To quantify the viral reservoir within intestinal tissues-which exhibit the greatest loss of $\mathrm{CD}^{+}{ }^{+} \mathrm{T}$ cells after infection-we sorted and measured cellassociated viral DNA within colonic, $\mathrm{CD} 4{ }^{+} \mathrm{TM}$ cells $\left(\mathrm{CD} 95^{+}\right)$ at necropsy. Despite a trend for higher cell-associated viral DNA in IL-21- and probiotic-supplemented animals, no significant differences were noted in residual viral DNA between ARV-only $(0.0188 \pm 0.00566$ copies per 100 cells; mean \pm s.e.m.) and IL-21- and probiotic-supplemented $(0.0364 \pm 0.0219)$ animals (Figure $2 \mathbf{b})$. Thus, probiotic and IL-21 supplementation does not appear to markedly alter virus production or infectivity within intestinal tissues in SIV-infected, ARV-treated PTMs.

\section{Improved clinical status in ARV-treated macaques supplemented with IL-21 and probiotics}

Effective ARV therapy significantly improves disease progression and clinical prognosis in chronically HIV-infected individuals; however, prolonged therapy remains fraught with non-AIDS-related co-morbidities, ${ }^{1,2}$ especially in situations in

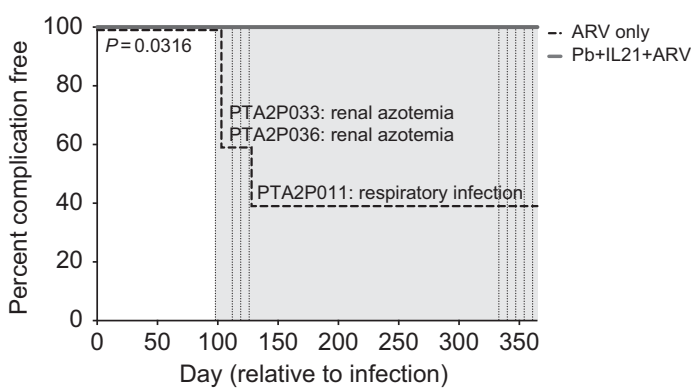

Figure 3 Interleukin (IL)-21 and probiotic therapy improves clinical status in antiretroviral (ARV)-treated pigtailed macaques (PTMs). Percent of animals remaining free from clinical complications over time. Initial complications per animal are noted alongside changes in the incidencefree curve, with ARV-only animals represented by the dashed line and supplemented animals by the solid line. Significance in the time to the first reported clinical complication between groups was assessed by the Mantel-Cox Log-Rank test.

which dysbiosis is likely to be present. ${ }^{26}$ To determine whether probiotic and IL-21 supplementation might improve the prognosis of our animals, we compared the incidence of clinical complications between our experimental groups, as diagnosed by positive bacterial culture or altered blood chemistries (Figure 3). Interestingly, although no overt complications were noted in the probiotic- and IL-21supplemented group, opportunistic infection (respiratory Enterobacter cloacae) and renal injury (azotemia as determined by elevated plasma levels of urea nitrogen or creatinine) were evident among three of the five ARV-only animals. ${ }^{27,28}$ Although low platelet counts have been shown to be associated with disease progression in SIV-infected PTM, ${ }^{29}$ we found similar counts between experimental groups throughout the study period (data not shown). Of animals that exhibited complications, all displayed subsequent instances of severe diarrhea with one animal requiring unplanned euthanasia, per protocol-specified humane clinical end points. To quantitatively assess disease progression, we 
compared the time to the first diagnosed clinical complication in ARV-only (median day 128 p.i.) and probiotic-, IL-21supplemented (no complication through study termination) animals and determined that supplementation was associated with a significant difference in the time to first complication $(P=0.0316$, Mantel-Cox). These results indicate that probiotic and IL-21 supplementation of ARV therapy decreased the incidence of non-AIDS morbidities.

\section{IL-21 and probiotic supplementation promote polyfunctional Th17 recovery in ARV-treated macaques}

In ARV-treated individuals and macaques, persistent microbial translocation and dysbiosis are associated with continued immune activation, thereby possibly exacerbating lentiviralmediated Th17 losses. ${ }^{16,17}$ As our previous findings indicated that probiotic supplementation of ARV therapy alone was unable to promote significant restoration of Th17 cells, we considered that additional supplementation with IL-21 might promote sustained restoration of these cells. We assessed the frequency of IL-17-expressing memory CD4 ${ }^{+}$T cells (Th17) longitudinally in the PBMCs, BAL, Jej, LN, and RB. Among $\mathrm{BAL}, \mathrm{LN}$, and PBMC CD4 ${ }^{+} \mathrm{T}$ cells, probiotic- and IL-21supplemented animals exhibited no significant differences in Th17 frequencies as compared with ARV-only controls or as compared with the pre-treatment time point of day 84 p.i. (data not shown). Indeed, preferential loss of Th17 within chronically $\mathrm{HIV} / \mathrm{SIV}$-infected individuals is not observed within these specific anatomic sites. ${ }^{7,30}$ However, modest to significant improvements in the frequencies of intestinal Th17 cells were observed in supplemented animals as compared with the pretreatment baseline time point (Figure $\mathbf{4 a}$ and $\mathbf{b}$ and Supplementary Figure S3A). Coupled with increased intestinal $\mathrm{CD}^{+}{ }^{+}$T-cell frequencies (Supplementary Figure S2), the improved relative frequencies of intestinal Th17 cells led to significantly improved absolute frequencies of intestinal Th17 cells (\% IL-17-expressing CD4 ${ }^{+}$TM of CD3 ${ }^{+} \mathrm{T}$ cells). We observed increases in the absolute frequency of Jej Th17 cells from day 84 p.i. to days 274 and 365 p.i. $(P=0.016,0.031)$ and in rectal Th17 cells from day 84 p.i. to days 126,274 , and 365 p.i. $(P=0.031,0.031,0.016$; Figure $4 \mathbf{a}$ and $\mathbf{b})$ within the probiotic- and IL-21-supplemented animals. No significant differences were evident in ARV-only controls (Supplementary Table S1). Furthermore, at day 182 p.i. (98 days post-intervention), ARV-only controls exhibited a significantly lower absolute frequency of Jej Th17 cells as compared with probiotic- and IL-21-supplemented animals $(P=0.0286)$.

Superior control of disease progression in HIV-infected individuals has been attributed to improved functionality (multiple cytokine expression, or polyfunctionality) among T cells. ${ }^{31-33}$ As such, we further investigated the frequency of intestinal, polyfunctional Th17 cells-as assessed here by the additional expression of IL-2, IL-22, or tumor necrosis factor- $\alpha$-in our animals. Although no significant differences were observed between groups in the expression of individual cytokines (data not shown), significant differences in the relative frequency of polyfunctional Jej Th17 cells were noted exclusively among probiotic- and IL-21-supplemented animals at necropsy as compared with days 84 and 274 p.i. $(P=0.031$, 0.031; Supplementary Figure S3B, Supplementary Table S1). As compared with ARV-only controls, the relative frequencies of polyfunctional Th17 cells were lower in supplemented animals at day 274 p.i. within the Jej $(P=0.033)$ and RB $(P=0.032)$ but comparable at all other time points. However, an examination of absolute intestinal polyfunctional Th17 frequencies revealed significant increases specific to probioticand IL-21-supplemented animals after the initiation of therapy (Figure 4c). Within Jej tissues, significant differences in the absolute frequencies of polyfunctional Th17 cells were observed from day 84 p.i. to days 274 and 365 p.i. $(P=0.031,0.031)$ in supplemented animals, in whom a significantly higher absolute frequency of polyfunctional Th17 cells was observed as compared with ARV-only controls at day 182 p.i. $(P=0.029)$. As compared with day 84 p.i., differences were additionally observed among probiotic- and IL-21-supplemented animals in the absolute frequency of RB polyfunctional Th17 cells at days 126,274 , and 365 p.i. $(P=0.031$, all $)$. No significant differences as compared with baseline were observed among the ARV-only controls at any time point (Supplementary Table S1). Thus, probiotic and IL-21 supplementation of SIV-infected, ARV-treated macaques led to a significant improvement in the absolute frequencies of intestinal Th17 cells and in turn, the absolute frequencies of polyfunctional Th17 cells.

As IL-21 similarly promotes the differentiation and maintenance of IL-17-expressing memory CD ${ }^{+}{ }^{+}$cells $\left(T_{C} 17\right)$, we additionally examined the longitudinal relative frequencies of $\mathrm{T}_{\mathrm{C}} 17$ cells. No significant differences between experimental groups were noted at any time point in any tissue examined (Supplementary Figure S3C and data not shown). However, a significant improvement in Jej $\mathrm{T}_{\mathrm{C}} 17$ cells was noted in the probiotic- and IL-21-supplemented animals at day 274 p.i. as compared with the pre-treatment time point $(P=0.0469)$ - no differences were noted in relative $\mathrm{RB} \mathrm{T}_{\mathrm{C}} 17$ frequencies between or among groups. We similarly examined the relative frequencies of polyfunctional $\mathrm{T}_{\mathrm{C}} 17$ cells. No differences were noted between experimental groups and, again, significant changes were limited to Jej $\mathrm{T}_{\mathrm{C}} 17$ cells in the probiotic- and IL-21-supplemented animals (Supplementary Figure S3D). Significantly higher relative frequencies of polyfunctional, Jej $\mathrm{T}_{\mathrm{C}} 17$ cells were noted in these animals at days 274 and 365 p.i. as compared with day 84 p.i. $(P=0.031,0.031)$. These data indicate that probiotic- and IL-21-supplementation of ARV therapy promotes a recovery of Th17 and $\mathrm{T}_{\mathrm{C}} 17$ cells during chronic SIV infection in macaques.

\section{IL-21 and probiotic supplementation reduce microbial translocation and dysbiosis in ARV-treated macaques}

Persistent immune activation, Th17 depletion, residual microbial translocation, and dysbiosis form a synergistic axis in ARV-treated individuals that likely results in the increased morbidities and mortality observed in ARV-treated subjects. ${ }^{26}$ 

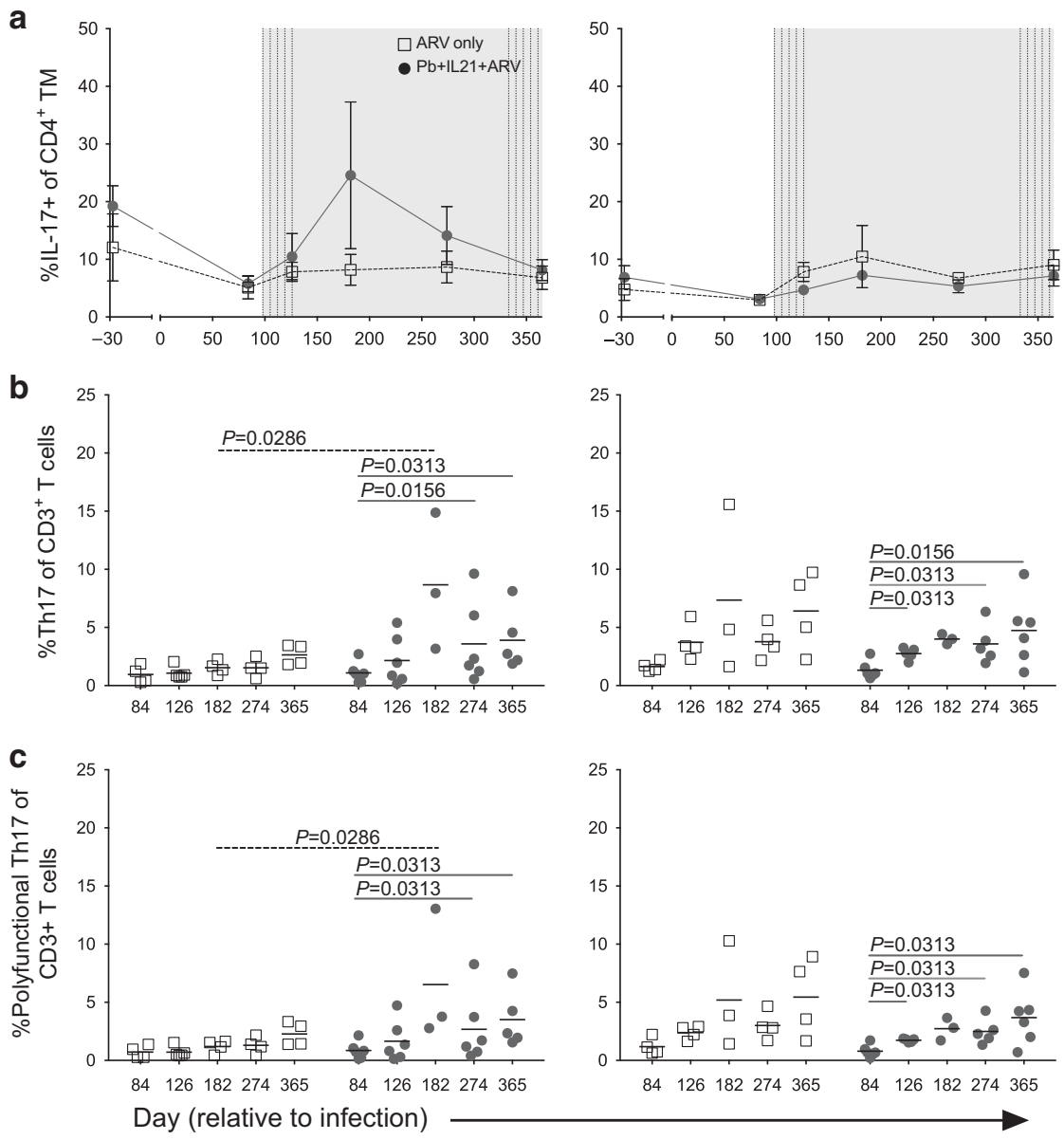

Jej

RB

Figure 4 Interleukin (IL)-21 and probiotic therapy promotes the absolute expansion of polyfunctional Th17 cells in ARV-treated PTMs. (a) Longitudinal mean ( \pm s.e.m.) percent IL-17 ${ }^{+}$of CD4 ${ }^{+}$TM (Th17) from the jejunal (Jej; left) and rectal biopsies (RB; right). (b) Absolute percent Th17 (that is, the percent of IL- $17^{+} \mathrm{CD} 4^{+} \mathrm{TM}$ found within $\mathrm{CD}^{+}{ }^{+}$T cells) from the Jej and RB at the indicated days p.i. (c) Absolute percent polyfunctional Th17 from the Jej and RB at the indicated days p.i. Line through samples represents mean; lines above samples span time points of significance. Significance within groups was assessed by Wilcoxon Signed-Rank test. Significance across groups was assessed by Mann-Whitney $U$-test.

As our results indicate that probiotic and IL-21 supplementation of ARVs promotes the recovery of intestinal Th17 cells (Figure 4), tempers some markers of immune activation (Supplementary Figure S1), and results in better clinical outcomes (Figure 3), we next considered the possibility that such treatment might further be associated with a reduction in microbial translocation and dysbiosis. We assessed levels of microbial translocation in our animals by immunohistochemical staining for Escherichia coli antigens in the colonic lamina propria, mesenteric LN paracortex, and liver tissue from samples obtained at necropsy. E. coli antigens were evident in all tissues and only within the liver of probiotic- and IL-21supplemented animals was there a trend for lower levels of E. coli staining $(P=0.0556$; Supplementary Figure $\mathbf{S 4} \mathbf{A}+\mathbf{B})$.

To assess whether probiotic and IL-21 supplementation influence the composition of microbial communities, we measured relative levels of commensal Bifidobacterium and Lactobacillus 16S DNA in the stool and rectal tissues of our experimental animals. No significant differences were observed in the ARV-only animals as compared with baseline with regards to Bifidobacterium levels $(P=0.0625$, all time points), but these animals exhibited significantly lower frequencies of Bifidobacterium as compared with probioticand IL-21-supplemented animals at day 154 p.i. $(P=0.0333$; Figure 5a). Importantly, probiotic- and IL-21-treated animals exhibited significant increases in the relative frequency of Bifidobacterium species as compared with the pre-treatment time point at days 154 and 365 p.i. $(P=0.0156,0.0313)$. Supplemented animals additionally exhibited significant increases in Lactobacillus species at day 154 p.i. as compared with the pre-treatment time point $(P=0.0156$; Figure 5b) -no significant differences were noted as compared with or among ARV-only animals ( $P=0.0625$, all time points). Thus, probiotic and IL-21 supplementation of ARV therapy during chronic SIV infection promoted a reduction in distal site microbial translocation as well as an increase in the level of probiotic species within the gastrointestinal tract, reflecting a significant improvement in disease progression. 
a

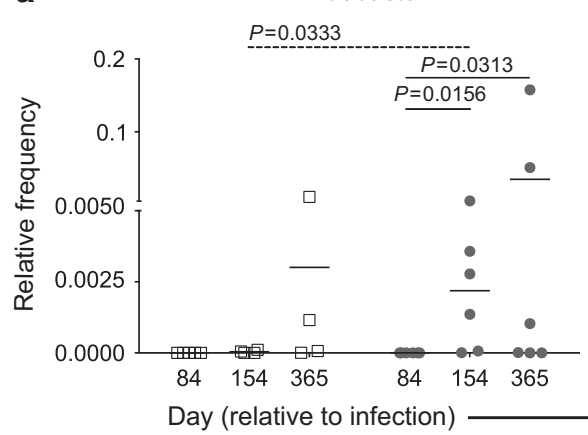

C

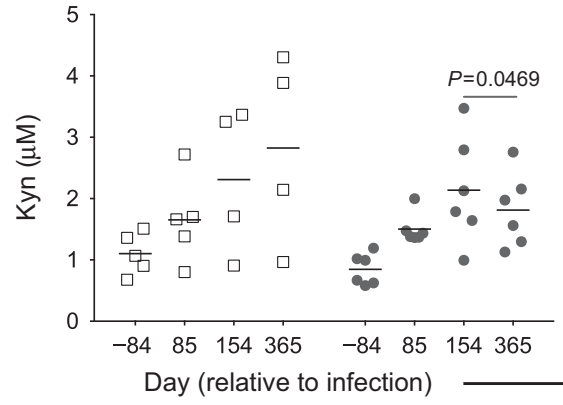

b

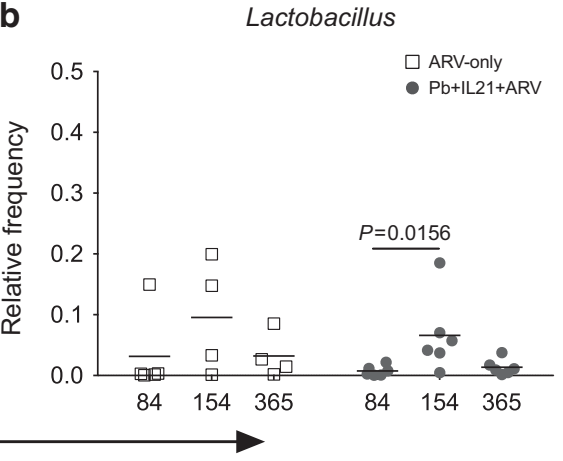

d

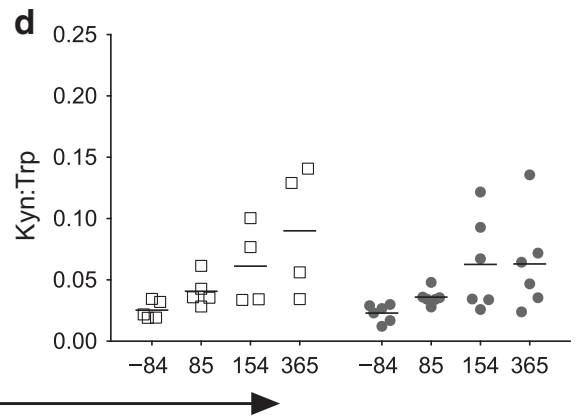

Figure 5 Interleukin (IL)-21 and probiotic therapy improve commensal colonization and reduce circulating kynurenine (Kyn). (a, b) Relative frequency of Bifidobacter (a) and Lactobacillus (b) 16S DNA of total 16S DNA in rectal tissues at the indicated time points. (c) Concentration of plasma Kyn ( $\mu \mathrm{m}$ ) at the indicated days p.i. (d) Ratio of plasma Kyn to Trp concentrations at the indicated days p.i. Line through samples represents mean; lines above samples span time points of significance. Significance within groups was assessed by Wilcoxon Signed-Rank test. Significance across groups was assessed by Mann-Whitney U-test.

\section{Reduced indoleamine-2,3-dioxygenase (IDO) activity in IL-21-, probiotic-, and ARV-treated macaques}

The persistent depletion of Th17 cells in ARV-treated, HIVinfected individuals correlates with elevated expression and activity of IDO, a cytokine-inducible, tryptophan (Trp)catabolizing enzyme. ${ }^{16,34}$ Trp metabolites are known to inhibit the generation and maintenance of Th17 cells, ${ }^{34}$ and persistently elevated IDO activity has been postulated to contribute to poor Th17 recovery in HIV-infected individuals ${ }^{16,34}$ while also predicting poor $\mathrm{CD} 4^{+} \mathrm{T}$-cell recovery and increased mortality. To determine whether probiotic and IL-21 supplementation reduced IDO expression and activity, we first quantified IDO expression by immunohistochemical staining in colon and mesenteric LN tissue samples obtained at necropsy. Tissue staining revealed no significant differences in mesenteric LN IDO between the two groups and only a trend for reduced colonic IDO in probiotic and IL-21 supplemented animals as compared with ARV controls $(P=0.0857$; Supplementary Figure S4C + D).We next considered whether Th17 recovery might correspond with a reduction in circulating levels of kynurenine (Kyn), the first immunoactive Trp catabolite generated in the IDO pathway. When we measured the concentration of plasma Kyn, we observed that whereas ARV-only animals exhibited a steady increase in Kyn throughout the study period, probiotic- and IL-21-supplemented animals displayed reduced Kyn from days 154 to 365 p.i. (Figure 5c). Although no significant differences were noted between experimental groups at the time points examined, significantly lower Kyn was apparent between these later time points within the supplemented group $(P=0.0469)$. To ascertain whether the observed Kyn levels might result from reduced IDO expression and/or activity, we additionally measured the ratio of plasma Kyn/Trp. Similar to the pattern observed for Kyn itself, although ARV-only animals exhibited a steady increase in plasma Kyn/Trp throughout the experimental period, probiotic- and IL-21-supplemented animals exhibited an increase only through the final examined prenecropsy time point, day 154 p.i. (Figure 5d). There was no significant difference between the final two time points, however, although supplemented animals exhibited a plateau in Kyn/Trp levels. Thus, probiotic and IL-21 supplementation of ARVs during chronic SIV infection is associated with a trend toward reduction in intestinal IDO and an apparent reduction in circulating levels of the Trp catabolite, Kyn.

\section{DISCUSSION}

ARV treatment significantly prolongs the length and quality of life of HIV-infected individuals; however, disease progression in these individuals remains evident even with decades of effective viral suppression. ${ }^{1,2}$ In treated HIV-infected individuals, morbidities and mortality have been attributed to a self-reinforcing cycle of microbial translocation and dysbiosis, immune activation, and a persistent loss of antimicrobial Th17 
cells. ${ }^{3,35}$ Although we have previously demonstrated that probiotic supplementation of ARVs in SIV-infected macaques promotes a restoration of intestinal $\mathrm{CD} 4{ }^{+} \mathrm{T}$ cells, ${ }^{18}$ in our prior study we did not observe significant Th17 expansion or was there a measurable impact on microbial translocation or on the composition of the gastrointestinal tract microbiome. Herein, we demonstrate that the supplementation of ARVs with probiotics and IL-21 promotes intestinal Th17 recovery, a reduction in measures of microbial translocation, and an enhanced microbiome. Importantly, our treatment regimen did not promote immune activation or a significant expansion of the viral reservoir. Although limited in size, these findings suggest that probiotic and IL-21 supplementation of ARV therapy should be further evaluated as a therapeutic in the treatment of non-AIDS-related co-morbidities.

The development of Th17 cells from naïve $\mathrm{CD} 4^{+}$T-cell precursors or $\mathrm{T}_{\text {regs }}$ is augmented by the autocrine production of IL-21. ${ }^{19-21}$ In this capacity, IL-21 enhances ROR $\gamma \mathrm{t}$ transcription, FOXP3 suppression, and the downstream transcription of IL-17 and additional Th17-associated cytokines such as IL-22. ${ }^{19,20}$ Importantly, IL-21 alone is insufficient to induce Th17 development, ${ }^{21}$ requiring additional signaling originating from the commensal microbiome. Thus, in murine models, although the presence of the commensal microbiome-specifically, segmented filamentous bacteria-is sufficient to drive the development of Th17 cells in vivo, ${ }^{13,14}$ an absence or blockade of IL-21 signaling diminishes Th17 differentiation. ${ }^{19,20}$ That the inclusion of IL-21 in our regimen promotes Th17 reconstitution (Figure 4) where probiotic supplementation of ARVs alone does $\operatorname{not}^{18}$ indicates that therapeutically provided probiotic species alone are either insufficient or incorrectly parlayed into the development of Th17 cells during chronic SIV infection. We believe that the prolonged regeneration of Th17 cells in our animals subsequent to IL-21 withdrawal ( $\sim 7$ months between early and late administration; Figure 1) suggests that IL-21 functioned directly at the level of $\mathrm{CD}^{+}{ }^{+} \mathrm{T}$ cells to establish a basal threshold of Th17 cells necessary for self-propagation. Although a direct assessment of circulating IL-21 was not performed, attainment of a theoretical threshold is further supported by the observation that late IL-21 administration did not augment already enhanced Th17 frequencies (Figure 4). To this end, it would be of significant interest to determine whether increasing the concentration of administered IL-21 or lengthening early IL-21 therapy might not augment or accelerate Th17 recovery beyond what we describe here. Although we cannot discount alternative roles for IL-21 in improving disease progression in our supplemented animals-such as altered functionality among IL-21-responsive B cells and NK cells ${ }^{36}$-the administration of IL-21 during acute SIV infection in macaques leads to a selective maintenance of Th17 cells that is strictly dependent on the continued administration of IL- $21,{ }^{23}$ arguing against an ancillary role for IL-21 in sustaining Th17 frequencies. An assessment of the effects of IL-21 supplementation to ARV therapy independent of probiotic administration will significantly further our understanding of the conditions required for the generation and maintenance of Th17 cells in treated chronic lentiviral infections.

Although de novo Th17 development from naïve $\mathrm{CD} 4^{+}$ T-cell precursors is largely reliant on the ability of APCs to integrate microbial sensing into pro-Th17 cytokine signaling, ${ }^{10,11}$ Th17 and $\mathrm{T}_{\text {reg }}$ cells exist as a transcriptional dichotomy, ${ }^{19,20,37,38}$ which may further be reciprocally programmed by aryl hydrocarbon receptor ligand signaling. ${ }^{34,39}$ As recent findings have demonstrated a role for the IDO-derived aryl hydrocarbon receptor-agonist Kyn in reducing the Th17/ $\mathrm{T}_{\text {reg }}$ during lentiviral infection, ${ }^{34}$ reduced plasma Kyn (Figure 5c) in our supplemented animals suggests that Th17 recovery may have included mechanisms adjunct to the direct influence of therapeutic IL-21. Given that species of the dysbiotic microbiota encode enzymatic homologues to primate Trp-catabolizing enzymes, ${ }^{16}$ we cannot discount the possibility that an IL-21-mediated recuperation of Th17 cells preempted a reduction of dysbiotic species and, hence, Kyn. Indeed, the prolonged recovery of commensal Bifidobacteria in our supplemented animals (Figure $\mathbf{5 b}$ ) in contrast to animals receiving probiotic supplementation alon $\mathrm{e}^{18}$ suggests a role for IL-21 in promoting the colonization of probiotic species. However, as a replete commensal microbiome is itself necessary for Th17 maintenance, ${ }^{14}$ prolonged Th17 recovery after IL-21 cessation may thus be dependent upon a reduction in the frequency of dysbiotic species.

Microbial translocation has been extensively described as a significant contributor to immune activation and disease progression in chronic lentiviral infections. ${ }^{3}$ In HIV-infected humans and SIV-infected non-human primates, the influence of microbial translocation on immune activation has been causally associated by the correlation of disseminated LPS with systemic interferon- $\alpha,{ }^{4,5}$ and formally demonstrated by the prevention of immune activation with acute use of the LPSsequestering drug sevelamer. ${ }^{40}$ Intriguingly, Th17 recovery (Figure 4) and improved disease progression (Figure 3) occurred independent of systemically reduced $E$. coli antigen (albeit a trend for reduced E. coli in the liver; Supplementary Figure $\mathbf{S 4 A}+\mathbf{B}$ ) or persistent immune activation (Supplementary Figure S1). In this regard, immune activation in our supplemented animals may reflect improved immune function rather than a manifestation of innate cell refractoriness or lymphocyte exhaustion. ${ }^{4,41,42}$ Indeed, as compared with ARVonly controls, supplemented animals exhibited control over rectal CD ${ }^{+}$T-cell proliferation (Supplementary Figure S1A) concomitant with improved $\mathrm{CD}^{+}{ }^{+}$T-cell reconstitution (Supplementary Figure S2), which is suggestive of homeostatic restoration. A more detailed assessment of immune function, particularly alterations in inflammatory biomarkers, will be necessary to address these hypotheses.

Increased mortality among HIV-infected individuals remains evident even with decades of effective viral suppression, ${ }^{2}$ especially in conditions when the IDO-mediated kynurenine pathway is induced and microbial dysbiosis may be present. ${ }^{26}$ Consistent with these observations in humans, co-morbidities were evident in our ARV-only animals 
where, despite continued viral suppression (Figure 2), three of five animals displayed repeated complications. That none of our supplemented animals displayed complications despite co-housing with the ARV-only controls is particularly notable given the propensity of PTMs toward microbial translocation and systemic immune activation, even in the absence of SIV infection. ${ }^{43}$ Although the recovery of Th17 cells in our supplemented animals (Figure 4) likely contributed to improved resistance to co-infection, ${ }^{8,9}$ an association between Th17 recovery and ARV tolerance is more tenuous. Indeed, ARV complications were reduced in animals supplemented with probiotics alone, where Th17 recovery was absent. ${ }^{18}$ Although the presence of commensal bacterial species has been demonstrated to improve the efficacy of anticancer therapeutics such as cyclophosphamide, ${ }^{44,45}$ the influence of probiotics and commensal species in mediating pharmaceutical toxicities has not been formally explored. A thorough characterization of the contributions of our individual therapeutic constituentsincluding individual probiotic species-to immune function in the presence and absence of ARVs will be necessary to fully uncover the mechanisms, which govern disease progression in treated chronic lentiviral infections.

In summary, we demonstrate that probiotic and IL-21 supplementation of ARV therapy in chronically SIV-infected macaques improves clinical outcomes, as manifested by a reduction in non-AIDS associated morbidities. Importantly, improved clinical outcomes were accompanied with an increase in the absolute frequency of intestinal, polyfunctional Th17 cells, and reduced measures of intestinal microbial dysbiosis. A larger, mechanistic dissection of the immune processes associated with improved disease progression in our supplemented animals will significantly inform the design of future therapeutics for the treatment of HIV-infected individuals.

\section{METHODS}

Animal infections and interventions. Eleven, healthy pigtail macaques (Macaca nemestrina) were accessed for this study and

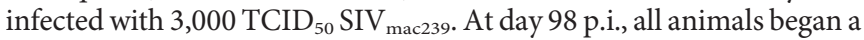
daily ARV regimen consisting of tenofovir and emtricitabine (30 $\mathrm{mg} \mathrm{kg}^{-1}$ at $120 \mathrm{mg} \mathrm{ml}^{-1}$ in PBS s.c. once-daily) and L'812 (120 mg oral, twice-daily) - tenofovir was reduced to $20 \mathrm{mg} \mathrm{kg}^{-1}$ after 30 days. Of these animals, 6 additionally received daily probiotic VSL\#3 (oral, two capsules daily) for the entirety of the study and two courses of rMamu IL-21-IgFc $\left(50 \mu \mathrm{g} \mathrm{kg}^{-1}\right.$ at $1 \mathrm{mg} \mathrm{ml}^{-1}$ in $\mathrm{H}_{2} \mathrm{O}$ s.c., 5 onceweekly doses) initiated at days 98 and 333 p.i. Animals were assigned to treatment groups to evenly distribute animals by weight and MHC genotype (Mane-A10) and were sampled as a mixed population. The National Institute of Allergy and Infectious Diseases Division of Intramural Research Animal Care and Use Program, as part of the National Institutes of Health Intramural Research Program, approved all of the experimental procedures (protocol LMM 12). Animals were killed according to the recommendations set forth by the American Veterinary Medical Association Guidelines on Euthanasia.

Sample collection. Blood, BAL, and biopsies from the colon, liver, lymph nodes, jejunum, and rectum were collected longitudinally or at necropsy. Biopsies were maintained in RPMI before processing. For longitudinal LN biopsies, axillary or inguinal LNs were locally exposed and excised by blunt dissection. For longitudinal RBs, fecal material was removed from the rectum and biopsies obtained with biopsy forceps. Jej biopsies were obtained by video-guided endoscopy. For BAL collection, silicone tubing was directed into the trachea with the assistance of a laryngoscope, whereupon warmed normal PBS or saline was instilled and subsequently aspirated for collection. Biopsies collected at necropsy were directly accessed immediately postmortem. Stool and luminal swabs were obtained as previously described. ${ }^{17}$ Plasma was isolated from blood by centrifugation. Mononuclear cells were isolated from blood by Ficoll gradient centrifugation and from BAL and tissue biopsies by straining/grinding samples through a $0.22-\mu \mathrm{m}$ cell strainer.

Determination of viral RNA and viral DNA. Plasma viral RNA levels were determined by quantitative reverse transcription-PCR (ABI Prism 7900 sequence detection system; Applied Biosystems, Waltham, MA). Viral RNA was reverse transcribed and cDNA amplified (45 cycles/default setting) with Ampli Taq Gold DNA polymerase (PCR core reagents kit; Perkin-Elmer/Roche, Waltham, MA) utilizing primer pairs corresponding to $\mathrm{SIV}_{\text {mac239 }}$ gag gene sequences (forward, nucleotides 1181-1208, and reverse, nucleotides 1338-1317). Cell-associated viral DNA was measured as previously. ${ }^{46}$ Briefly, quantitative PCR was performed on sorted, lysed cells using the Taq DNA polymerase kit (Invitrogen, Waltham, MA) and the $\mathrm{SIV}_{\text {mac239 }}$ forward primer 5'-GTCTGCGTCATYTGGTGCATTC-3', reverse primer $5^{\prime}$-CACTAGYTGTCTCTGCACTATRTGTTTTG- $3^{\prime}$, and probe sequence $5^{\prime}$-CTTCRTCAGTYTGTTTCACTTTCTCTTCTG CG- $3^{\prime}$. Results assessed below the limit of detection were normalized to the threshold cutoff.

Flow cytometry and immunophenotypic analysis. Polychromatic flow cytometry and cell sorting were performed on stained mononuclear cells utilizing the BD LSRFortessa and FACS Aria (BD Biosciences, Franklin Lakes, NJ), respectively, equipped with FACS DiVA software (BD Biosciences). The following antibodies were used for staining at predetermined concentrations: CD3 (clone SP34-2) AL700, HLADR (G46-6) APC-H7, IL-2 (MQ1-17H12) FITC, and Ki67 (B56) FITC from BD Biosciences; CD4 (OKT4) eFluor450, CD8 (SK1) PerCP-e710, IFNg (4S.B3) eFluor450, IL17A (eBio64DEC17) $\mathrm{PE}$, IL22 (IL22JOP) APC, and TNFa (MAb11) PE-Cy7 from eBioscience (San Diego, CA); CD28 (CD28.2) ECD from Beckman Coulter (Pasadena, CA); and CD95 (DX2) PE-Cy5 from BioLegend (San Diego, CA). Cell viability was assessed using the Live/Dead Aqua Fixable Dead Cell Stain (Invitrogen). Cells were permeabilized with Cytofix/Cytoperm (BD Biosciences) before intracellular staining. Acquired data were analyzed using FlowJo software (Treestar Ashland, OR). To quantify cell subset frequencies, we used a threshold cutoff of 100 cells of the parent population. For Th17 functional analyses, cytokine gates were established on a threshold cutoff of 100 $\mathrm{CD}^{+}{ }^{+}$or $\mathrm{CD}^{+}{ }^{+} \mathrm{TM}$ and Boolean gates utilized to ascertain frequencies-populations not meeting this threshold were not considered for analysis.

Immunohistochemical and quantitative image analysis. Immunohistochemical for rabbit polyclonal anti-E. coli (Cat. \# B0357; Dako, Santa Clara, CA) and rabbit monoclonal anti-IDO (Millipore, Billerica, MA; clone EPR1230Y) was performed using a biotin-free polymer approach (Rabbit Polink-1 for E. coli and Mouse Polink-2 for IDO, Golden Bridge International, Bothell, WA) on 5-mm tissue sections mounted on glass slides, which were dewaxed and rehydrated with double-distilled $\mathrm{H}_{2} \mathrm{O}$. Antigen retrieval was performed by heating slides in $\times 1$ DIVA Decloacker reagent (Biocare Medical, Concord, CA) or $0.01 \%$ citraconic anhydride containing $0.05 \%$ Tween-20 in a pressure cooker $\left(122^{\circ} \mathrm{C}\right.$ for $\left.30 \mathrm{~s}\right)$. Slides were stained with optimal conditions determined empirically on an IntelliPATH autostainer (Biocare Medical) that consisted of a blocking step using blocking buffer (tris-buffered saline with $0.05 \%$ Tween- 20 and $0.5 \%$ casein) for $10 \mathrm{~min}$ and an endogenous peroxidase block using $1.5 \%(\mathrm{v} / \mathrm{v}) \mathrm{H}_{2} \mathrm{O}_{2}$ in TBS ( $\mathrm{pH}$ 7.4) for $10 \mathrm{~min}$. 
Primary antibodies were diluted 1:5,000 in blocking buffer and incubated for $1 \mathrm{~h}$ at room temperature. Tissue sections were washed, and either Rabbit Polink-1 or Polink-2 staining systems (Golden Bridge International) were applied for $30 \mathrm{~min}$ at room temperature. Sections were developed with Impact 3,3'-diaminobenzidine (Vector Laboratories, Burlingame, CA), counterstained with hematoxylin and mounted in Permount (Fisher Scientific, Hampton, NH). All stained slides were scanned at high magnification $(\times 400)$ using the Aperio AT2 System (Leica Biosystems, Wetzlar, Germany) yielding high-resolution data for the entire tissue section. Representative high-magnification $(400 \times)$ images were acquired from these whole-tissue scans.

Quantification of systemic inflammatory markers. Concentrations of Kyn and Trp were quantified from plasma by liquid-chromatography-tandem mass spectrometry, as previously described. ${ }^{34}$ Levels of LPS-binding protein and sCD14 were assessed from plasma using commercially available ELISA kits (Antibodies Online, Atlanta, GA and $\mathrm{R} \& \mathrm{D}$, Minneapolis, $\mathrm{MN}$, respectively), according to the manufacturers' protocols.

Assessment of bacterial 16S DNA. 16S DNA was isolated and quantified from stool or from luminal swabs as previously described. ${ }^{17}$

Statistics. Statistical analyses were performed using Prism (v6.0; GraphPad Software, La Jolla, CA). The Mann-Whitney $U$-test was used for comparisons between groups; the Wilcoxon Signed-Rank test for within groups. The Mantel-Cox test was used to assess disease progression as measured by time to the first presentation of health complication. Averaged data are presented as arithmetic mean \pm s.e.m. $P$ values less than 0.05 was considered significant.

SUPPLEMENTARY MATERIAL is linked to the online version of the paper at http://www.nature.com/mi

\section{ACKNOWLEDGMENTS}

We thank F. Villinger (Emory University) for providing the IL-21; D. Hazuda (Gilead), M. Miller (Merck), and J. Lifson (NCl) for providing the ARVs; C. de Simon (Sigma Tau Pharmaceuticals) for providing the VSL\#3; H. Cronise, J. Swerczek, and R. Herbert for veterinary assistance; and the NIAID LMM-Core for assessing viral loads. Funding for this study was provided in part by the Division of Intramural Research/NIAID/NIH. This project has been funded in part with federal funds from the National Cancer Institute, National Institutes of Health, under Contract No. HHSN261200800001E to JDE. The content of this publication does not necessarily reflect the views or policies of DHHS, or does the mention of trade names, commercial products, or organizations imply endorsement by the US Government.

\section{DISCLOSURE}

The authors declared no conflict of interest.

c 2016 Society for Mucosal Immunology

\section{REFERENCES}

1. Lederman, M.M., Funderburg, N.T., Sekaly, R.P., Klatt, N.R. \& Hunt, P.W. Residual immune dysregulation syndrome in treated HIV infection. Adv. Immunol. 119, 51-83 (2013).

2. Volberding, P.A. \& Deeks, S.G. Antiretroviral therapy and management of HIV infection. Lancet 376, 49-62 (2010).

3. Marchetti, G., Tincati, C. \& Silvestri, G. Microbial translocation in the pathogenesis of HIV infection and AIDS. Clin. Microbiol. Rev. 26, 2-18 (2013).

4. Brenchley, J.M. et al. Microbial translocation is a cause of systemic immune activation in chronic HIV infection. Nat. Med 12, 1365-1371 (2006).

5. Estes, J.D. et al. Damaged intestinal epithelial integrity linked to microbial translocation in pathogenic simian immunodeficiency virus infections. PLoS Pathog. 6, e1001052 (2010).
6. Favre, D. et al. Critical loss of the balance between Th17 and T regulatory cell populations in pathogenic SIV infection. PLoS Pathog 5, e1000295 (2009).

7. Brenchley, J.M. et al. Differential Th17 CD4 T-cell depletion in pathogenic and nonpathogenic lentiviral infections. Blood 112, 2826-2835 (2008).

8. Raffatellu, M. et al. Simian immunodeficiency virus-induced mucosal interleukin-17 deficiency promotes Salmonella dissemination from the gut. Nat. Med 14, 421-428 (2008).

9. Peck, A. \& Mellins, E.D. Precarious balance: Th17 cells in host defense. Infect. Immun. 78, 32-38 (2010).

10. Veldhoen, M., Hocking, R.J., Atkins, C.J., Locksley, R.M. \& Stockinger, B. TGFbeta in the context of an inflammatory cytokine milieu supports de novo differentiation of IL-17-producing T cells. Immunity 24, 179-189 (2006).

11. Goto, Y. et al. Segmented filamentous bacteria antigens presented by intestinal dendritic cells drive mucosal Th17 cell differentiation. Immunity 40, 594-607 (2014).

12. Yang, Y. et al. Focused specificity of intestinal $\mathrm{TH} 17$ cells towards commensal bacterial antigens. Nature 510, 152-156 (2014).

13. Ivanov, II et al. Induction of intestinal Th17 cells by segmented filamentous bacteria. Cell 139, 485-498 (2009).

14. Ivanov, II et al. Specific microbiota direct the differentiation of IL-17producing T-helper cells in the mucosa of the small intestine. Cell Host Microbe 4, 337-349 (2008).

15. Littman, D.R. \& Pamer, E.G. Role of the commensal microbiota in normal and pathogenic host immune responses. Cell Host Microbe 10, 311-323 (2011).

16. Vujkovic-Cvijin, I. et al. Dysbiosis of the gut microbiota is associated with HIV disease progression and tryptophan catabolism. Sci. Trans. Med 5, 193ra191 (2013).

17. Klase, Z. et al. Dysbiotic bacteria translocate in progressive SIV infection. Mucosal Immunol. advance online publication, 14 January 2015; doi: 10.1038/mi.2014.128 (2015).

18. Klatt, N.R. et al. Probiotic/prebiotic supplementation of antiretrovirals improves gastrointestinal immunity in SIV-infected macaques. J. Clin. Invest 123, 903-907 (2013).

19. Korn, T. et al. IL-21 initiates an alternative pathway to induce proinflammatory $\mathrm{T}(\mathrm{H}) 17$ cells. Nature 448, 484-487 (2007).

20. Nurieva, R. et al. Essential autocrine regulation by IL-21 in the generation of inflammatory T cells. Nature 448, 480-483 (2007).

21. Yeste, A. et al. IL-21 induces IL-22 production in CD4 + T cells. Nat. Commun 5, 3753 (2014).

22. Micci, L. et al. Paucity of IL-21-producing CD4(+) Tcells is associated with Th17 cell depletion in SIV infection of rhesus macaques. Blood 120, 39253935 (2012).

23. Pallikkuth, S. et al. Maintenance of intestinal Th17 cells and reduced microbial translocation in SIV-infected rhesus macaques treated with interleukin (IL)-21. PLoS Pathog 9, e1003471 (2013).

24. Ma, H.L. et al. IL-21 activates both innate and adaptive immunity to generate potent antitumor responses that require perforin but are independent of IFN-gamma. J. Immunol 171, 608-615 (2003).

25. Pallikkuth, S. et al. Interleukin-21 administration to rhesus macaques chronically infected with simian immunodeficiency virus increases cytotoxic effector molecules in Tcells and NK cells and enhances B cell function without increasing immune activation or viral replication. Vaccine 29, 92299238 (2011).

26. Byakwaga, H. et al. The kynurenine pathway of tryptophan catabolism, CD4 + T-cell recovery, and mortality among HIV-infected Ugandans initiating antiretroviral therapy. J. Infect. Dis 210, 383-391 (2014).

27. Gaston, M.A. Enterobacter: an emerging nosocomial pathogen. J. Hosp. Infect 11, 197-208 (1988).

28. Perazella, M.A. Acute renal failure in HIV-infected patients: a brief review of common causes. Am. J. Med. Sci 319, 385-391 (2000).

29. Alcantara, S. et al. Thrombocytopenia is strongly associated with simian AIDS in pigtail macaques. J. Acquir. Immune Defic. Syndr 51, 374-379 (2009).

30. Klatt, N.R. et al. Loss of mucosal CD103 + DCs and IL-17 + and IL-22 + lymphocytes is associated with mucosal damage in SIV infection. Mucosal Immunol 5, 646-657 (2012). 
31. Almeida, J.R. et al. Superior control of HIV-1 replication by CD8 + Tcells is reflected by their avidity, polyfunctionality, and clonal turnover. J. Exp. Med 204, 2473-2485 (2007).

32. Betts, M.R. et al. HIV nonprogressors preferentially maintain highly functional HIV-specific CD8 + T cells. Blood 107, 4781-4789 (2006).

33. Brenchley, J.M. et al. High frequencies of polyfunctional HIV-specific Tcells are associated with preservation of mucosal CD4 Tcells in bronchoalveolar lavage. Mucosal Immunol 1, 49-58 (2008).

34. Favre, D. et al. Tryptophan catabolism by indoleamine 2,3-dioxygenase 1 alters the balance of TH17 to regulatory Tcells in HIV disease. Sci. Transl. Med 2, 32 ra36 (2010).

35. Kanwar, B., Favre, D. \& McCune, J.M. Th17 and regulatory T cells: implications for AIDS pathogenesis. Curr. Opin. HIV AIDS 5, 151-157 (2010).

36. Spolski, R. \& Leonard, W.J. Interleukin-21: a double-edged sword with therapeutic potential. Nat. Rev. Drug Discov 13, 379-395 (2014).

37. Bettelli, E. et al. Reciprocal developmental pathways for the generation of pathogenic effector TH17 and regulatory T cells. Nature 441, 235-238 (2006).

38. Zhou, L. et al. TGF-beta-induced Foxp3 inhibits $T(H) 17$ cell differentiation by antagonizing RORgammat function. Nature 453, 236-240 (2008).
39. Quintana, F.J. et al. Control of $T($ reg $)$ and $T(H) 17$ cell differentiation by the aryl hydrocarbon receptor. Nature 453, 65-71 (2008).

40. Kristoff, J. et al. Early microbial translocation blockade reduces SIV-mediated inflammation and viral replication. J. Clin. Invest 124, 2802-2806 (2014).

41. Lichterfeld, M. et al. Loss of HIV-1-specific CD8 + T cell proliferation after acute HIV-1 infection and restoration by vaccine-induced HIV-1-specific CD4 + T cells. J. Exp. Med 200, 701-712 (2004).

42. Appay, V. et al. HIV-specific CD8(+) T cells produce antiviral cytokines but are impaired in cytolytic function. J. Exp. Med 192, 63-75 (2000).

43. Klatt, N.R. et al. Compromised gastrointestinal integrity in pigtail macaques is associated with increased microbial translocation, immune activation, and IL-17 production in the absence of SIV infection. Mucosal Immuno/ 3, 387-398 (2010).

44. Viaud, S. et al. The intestinal microbiota modulates the anticancer immune effects of cyclophosphamide. Science 342, 971-976 (2013).

45. lida, $\mathrm{N}$. et al. Commensal bacteria control cancer response to therapy by modulating the tumor microenvironment. Science $\mathbf{3 4 2}$, 967-970 (2013).

46. Brenchley, J.M. et al. Differential infection patterns of CD4 + T cells and lymphoid tissue viral burden distinguish progressive and nonprogressive lentiviral infections. Blood 120, 4172-4181 (2012). 